(2) Open Access Full Text Article

\title{
Apatinib to combat EGFR-TKI resistance in an advanced non-small cell lung cancer patient with unknown EGFR status: a case report
}

This article was published in the following Dove Press journal:

OncoTargets and Therapy

26 April 2017

Number of times this article has been viewed

\author{
Yanmei Peng' \\ Huijuan Cui ${ }^{2}$ \\ Zhe Liu $^{3}$ \\ Daiwei Liu' \\ Fan Liu' \\ Yazhong Song' \\ Hua Duan' \\ Yuqin Qiu' \\ Qiang $\mathrm{Li}^{\prime}$ \\ 'Department of China-Japan \\ Friendship Hospital, Beijing University \\ of Chinese Medicine, ${ }^{2}$ Department \\ of Oncology, China-Japan Friendship \\ Hospital, Chaoyang, ${ }^{3}$ Department of \\ Oncology, Beijing Chest Hospital, \\ Tongzhou, Beijing, People's Republic \\ of China
}

Correspondence: Huijuan Cui

Department of Oncology, China-Japan

Friendship Hospital, No 2, Yinghua Dong

Jie, Chaoyang District, Beijing 100029,

People's Republic of China

Tel +86 I39 II8350I8

Email cuihj1963@sina.com
Abstract: Lung adenocarcinoma is the most common pathological pattern of lung cancer. During the past decades, a number of targeted agents have been explored to treat advanced lung adenocarcinoma. In the present clinical practice, antagonists of the epidermal growth factor receptor (EGFR) and vascular endothelial growth factor (VEGF)-directed therapies are widely used. In the former category, the agent erlotinib (tyrosine kinase inhibitor) has shown obvious advantages over cytotoxic therapy. Anti-VEGF therapy bevacizumab used for lung adenocarcinoma was recommended in NCCN Clinical Practice Guidelines in Oncology (NCCN Guidelines) as first-line therapy. Similarly, apatinib is speculated to response by selectively inhibiting the vascular endothelial growth factor receptor-2. The patient with unknown EGFR status benefited 5-month progressive free survival (PFS) from erlotinib, and then another 5.1-month PFS with combined treatment of apatinib, which suggested a new option for lung adenocarcinoma. However, when dabigatran was used to cancer-related venous thromboembolism during apatinib therapy, extensive subcutaneous bleeding occurred, warning us against the risks of bleeding. Besides, hypertension and anorexia were observed, causing dosage adjustment.

Keywords: NSCLC, epidermal growth factor receptor, vascular endothelial growth factor, venous thromboembolism, dabigatran

\section{Introduction}

Lung cancer is one of the leading causes of morbidity and mortality among cancer patients, posing a major threat to public health. ${ }^{1}$ In China, lung adenocarcinoma makes the maximum proportion. In Asian non-small cell lung cancer (NSCLC) patients, about 40\%-60\% epidermal growth factor receptor (EGFR) mutations are detected, for which anti-EGFR agents, erlotinib (F Hoffmann-La Roche Ltd, Basel, Switzerland), are proposed to use. Nowadays, EGFR-tyrosine kinase inhibitor (TKI) resistance is a popular research hotspot.

Apatinib (Hengrui Pharmaceutical Co. Ltd, Jiangsu, People's Republic of China) is a novel tyrosine kinase inhibitor that selectively inhibits the VEGF-2 and inhibits VEGF-mediated endothelial cell migration and proliferation, thus blocking new blood vessel formation in tumor tissue. ${ }^{2,3}$ Currently, phase II/III clinical trials in China have approved the indication of chemotherapy-refractory advanced metastatic gastric cancer. ${ }^{4,5}$ The phase I clinical trial showed a potential targeted treatment for advanced lung cancer, metastatic breast cancer, and advanced hepatocellular carcinoma. ${ }^{6}$

We here report a case of apatinib's response in an advanced NSCLC patient with EGFR-TKI resistance, who died of severe pneumonia with disease progression. As 
far as we know, this is the first report of the combination treatment with apatinib and erlotinib combating acquired EGFR-TKI resistance.

\section{Case report}

In August 2014, the male patient had a sudden chest distress and cough but without phlegm. Computed tomography (CT) of chest in Beijing Chest Hospital showed a mass in the left upper lung sized $\sim 41 \times 27 \mathrm{~mm}$, with segmented edge, metastasis in mediastinal 4R,7 regions and ipsilateral hilar lymph nodes, and right pulmonary pleura with micronodulaire. Cranial magnetic resonance imaging (MRI), abdominal CT, and bone scans were found normal. On September 28, 2014, adenocarcinoma was diagnosed by fine-needle aspiration biopsy under ultrasonic bronchoscope (Figure 1). EGFR test was unable to perform because of the small size of specimens. The patient was diagnosed with lung adenocarcinoma on the left upper lobe with poorly differentiated stage IIIb.

From October 3, 2014, to March 3, 2015, the patient was treated with the first-line chemotherapy (gemcitabine $2.2 \mathrm{~g}$ days 1 and 8,8 plus cisplatin $50 \mathrm{mg}$ days $1-3$, every 21 days) at Beijing Chest Hospital. The overall efficacy evaluation by thoracic CT was partial response (PR) after six therapy circles. The partial radiotherapy of mediastinum and mass in the left lobe was from April 20 to June 1, 2015, in Cancer Hospital, Chinese Academic of Medical Sciences, and the efficacy evaluation was PR. Left headache with blurred vision occurred on August 26, 2015. Cranial MRI showed metastasis in the left occipital lobe. Whole-brain radiotherapy and dehydration treatment eliminated the syndrome, and MRI showed edema, which was obviously alleviated, but the tumor in the left lobe was increased.

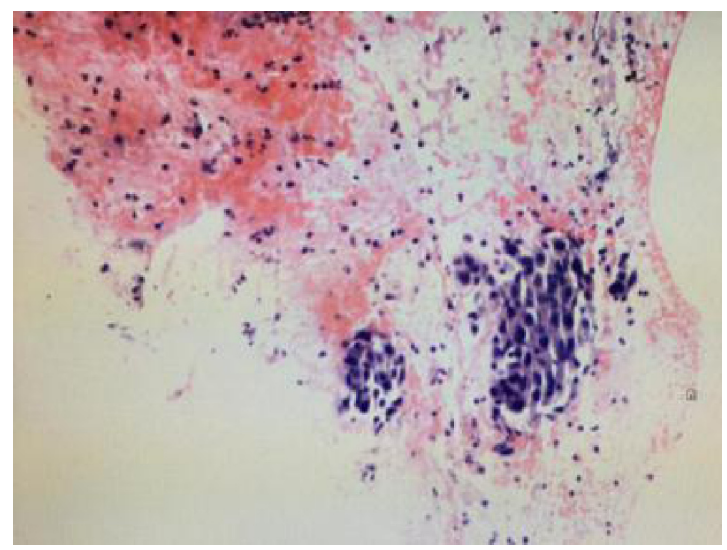

Figure I Tumor section staining with hematoxylin and eosin (400x).
Because of unbearable adverse events (AEs), especially gastrointestinal side effects, the patient asked for targeted therapy despite the unknown EGFR status. On September 30,2015 , the patient refused to receive re-biopsy and started taking erlotinib (150 mg/day) as the second-line therapy. Imageological examination demonstrated stable disease, verifying erlotinib was effective to the patient. However, cranial MRI on December 27, 2015, revealed edema aggravated. The patients received gamma knife radiation for brain metastasis in Beijing Tian Tan Hospital on December 30, 2015. Chest CT showed that the solid mass in the left upper lobe was slightly enlarged on February 26, 2016 (Figure 2A). A bone scan suggested bone metastases and the encephaledem slightly deteriorated (Figure 3A), a possible indication of progressive disease (PD) and erlotinib resistance. Combined use of erlotinib and apatinib (250 mg/day) was chosen as the third-line therapy from March 17, 2016 (Table 1) in China-Japan Friendship Hospital. After approximately a month's treatment, the chest CT on April 25, 2016, showed cavitation formed in the solid mass of the left lobe, and the solid mass was significantly reduced with stable mediastinal lymph node (Figure 2B). Cranial MRI on April 27, 2016, showed edema in the left occipital lobe alleviated (Figure 3B). Monthly imageological examination revealed the effectiveness of the combination treatment (Figures $2 \mathrm{C}-\mathrm{F}$ and $3 \mathrm{C}-\mathrm{E})$. Circulation tumor cell from the patient's plasma reported $3.87 \mathrm{FU} / 3 \mathrm{~mL}$ within normal limits on July 7 , 2016, whereas 30.1 FU/3 mL on August 29, 2016, beyond normal limits. The contrast might imply disease progression. Ultimately, another 5.1-month PFS was achieved and severe pneumonia took away the patient's life with disease progression on August 31, 2016.

The left upper limb was found swelling on July 5, 2016, and ultrasonography showed venous thromboembolism (VTE). Fraxiparine, low molecular weight heparin (LMWH) calcium, was applied to anticoagulant therapy for the monitoring coagulation function from July 8, 2016. On July 26, 2016, ultrasonography showed the sign of vascular recanalization and reduction of swelling. Considering the difficulty complying with INR monitoring, the patient requested medication on discharge, so dabigatran (Boehringer Ingelheim Pharmaceutical Co., Ingelheim, Germany), a new oral anticoagulants, was chosen. On August 21, 2016, the patient suddenly felt dizzy with spraying vomit, and extensive subcutaneous hemorrhage occurred. On August 25, the patient was hospitalized and treated with hemostasis and plasma supplement as the 
A

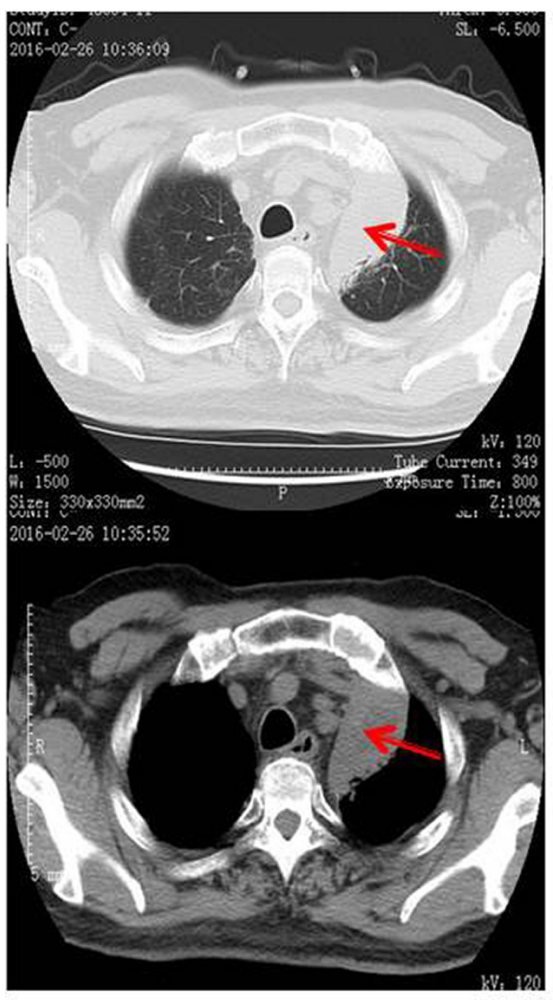

D

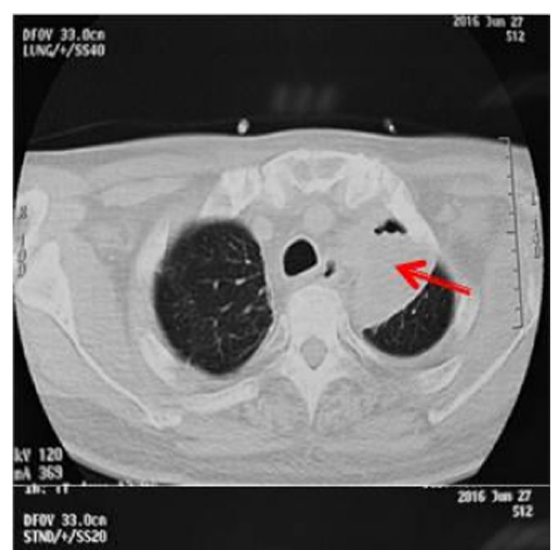

B

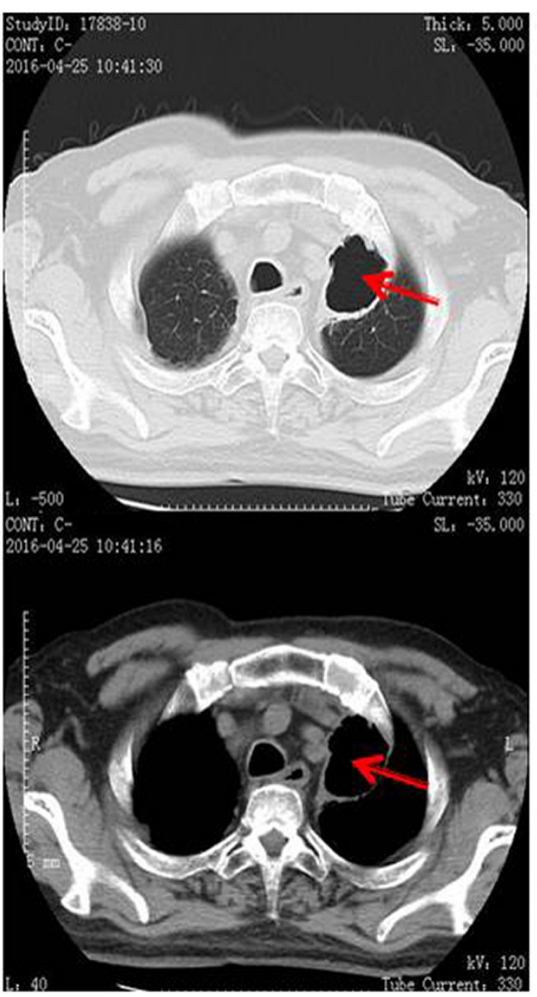

C
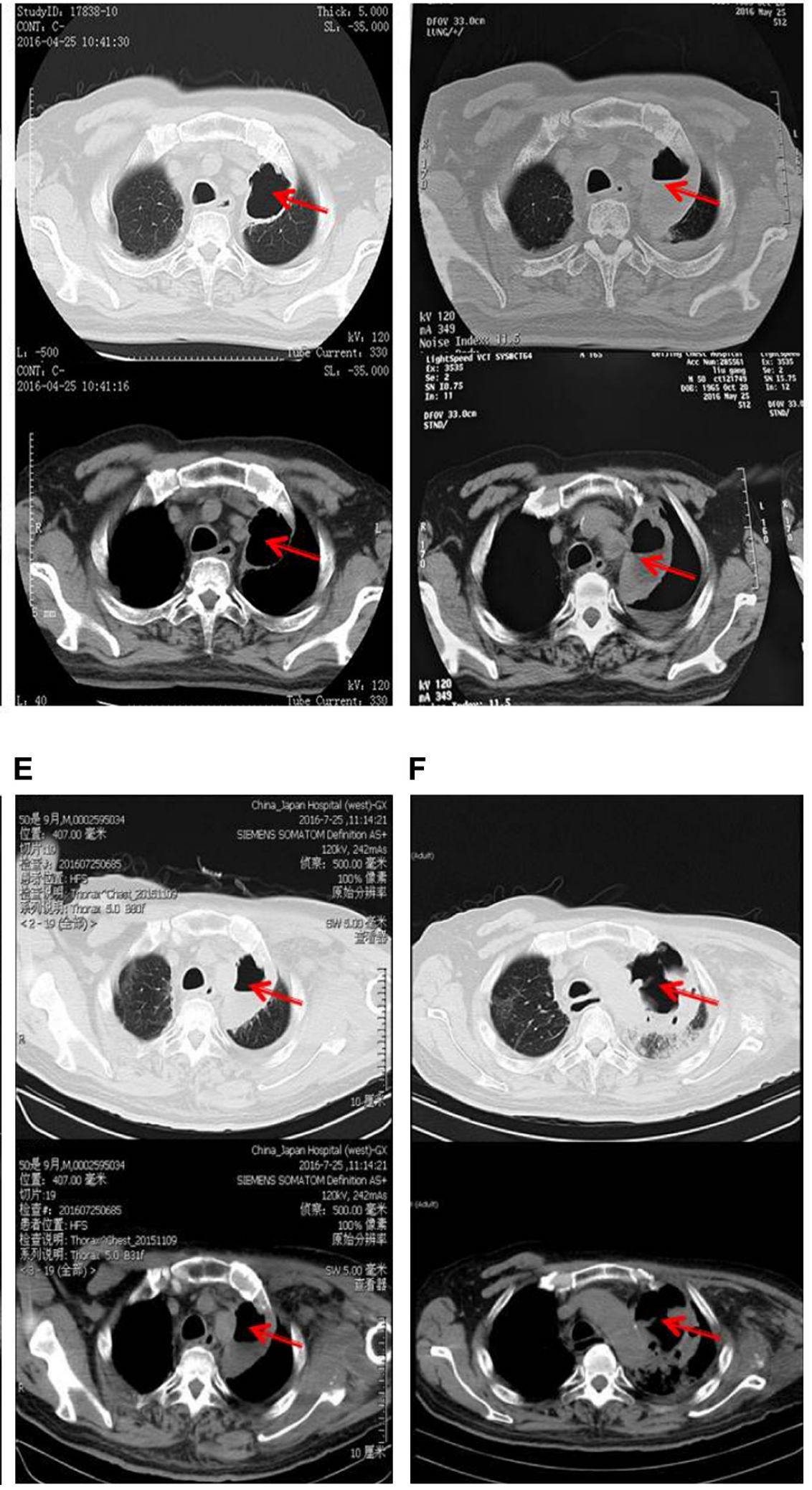

$\mathbf{F}$

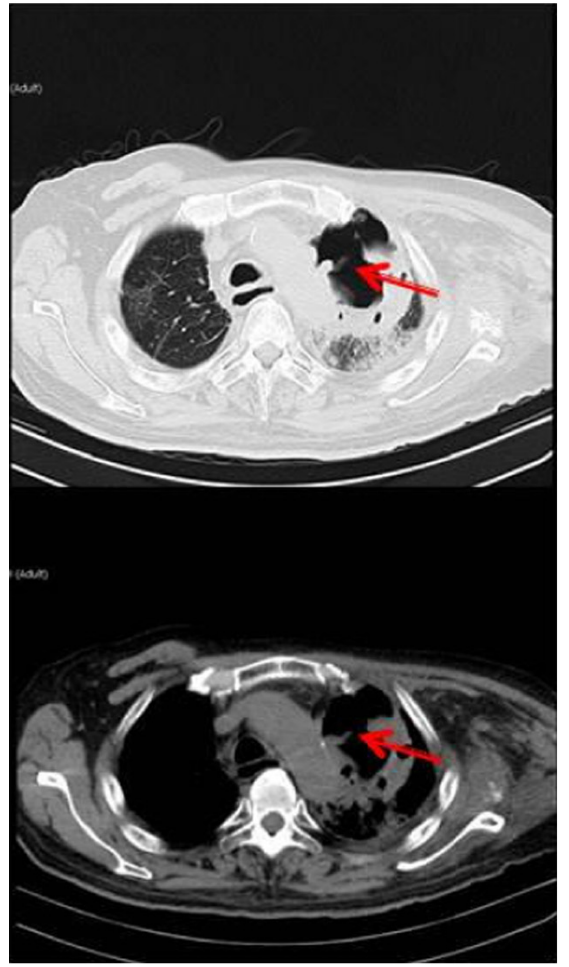

Figure 2 Thoracic computed tomography (CT) showing a mass in the left upper lobe before taking apatinib (A), an obvious cavitation in the mass (B), mass increasing after apatinib suspension for 6 days $(\mathbf{C})$, cavitation extending gradually with added dose of apatinib via monthly CT examination (D and E), and an overall increase of mass despite the larger cavitation $(\mathbf{F})$. The red arrows indicate specific region of tumor. 

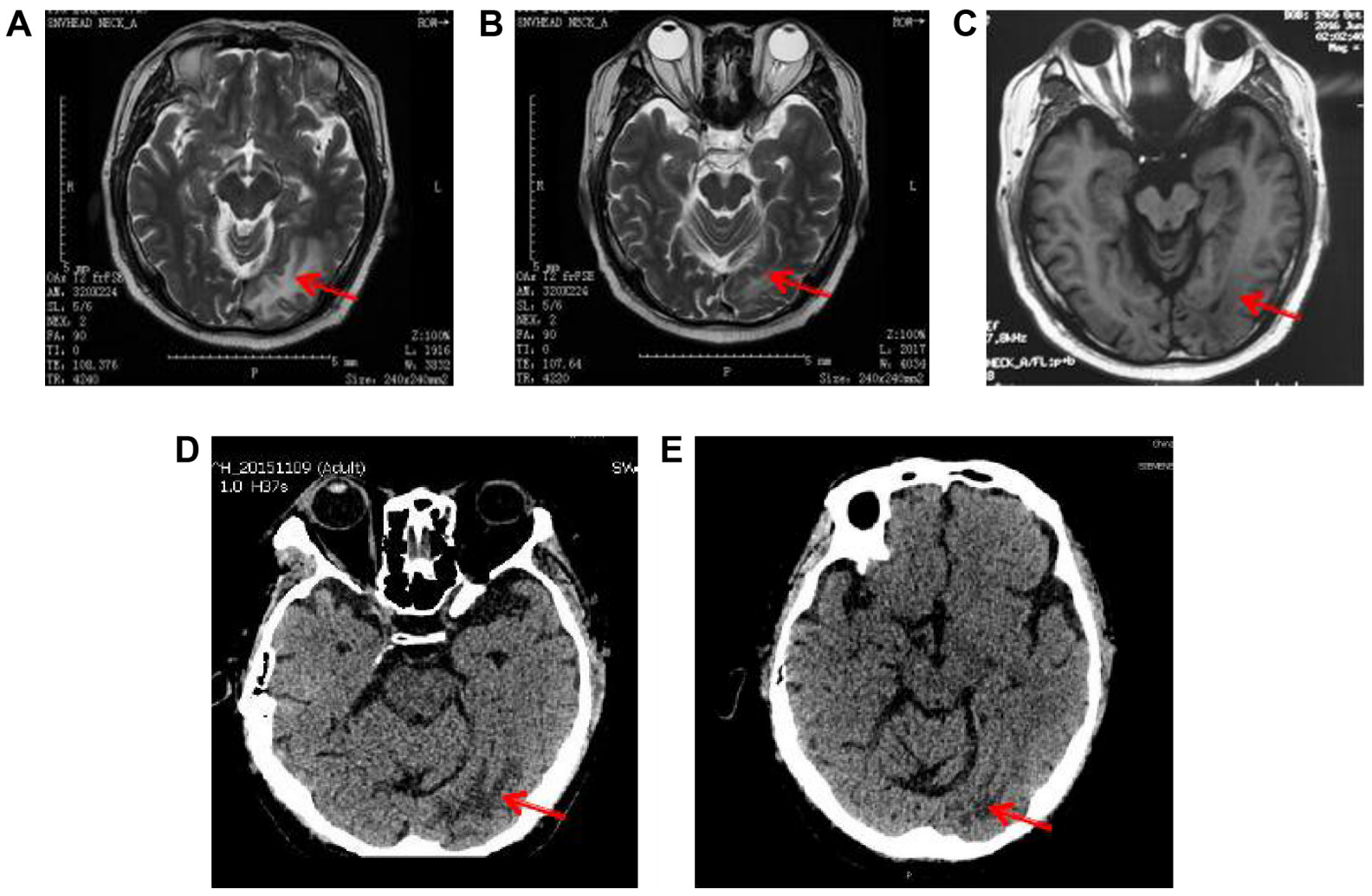

Figure 3 Cranial MRI showing the encephaledem in the occipital lobe of the head (A), encephaledem obviously alleviating (B), slightly worsening after suspension of apatinib for 6 days (C), the encephaledem basically under control as shown by CT every 2 months (D and $\mathbf{E})$.

Abbreviations: MRI, magnetic resonance imaging; $\mathrm{CT}$, computed tomography.

blood routine (Figure 4), and activated partial thromboplastin time (Figure 5) was monitored, stopping taking dabigatran. Ultrasonography presented no thrombus despite recurred swelling in the left upper limb (Figure 6) until his death. Written informed consent was obtained from the patient's wife for publication of the details and accompanying images for this study.

\section{Discussion}

The patient was initially diagnosed with left lung adenocarcinoma via pathology tests. Platinum-based doublets chemotherapy was recommended as the first-line therapy. Systemic chemotherapy and local radiotherapy were given to the patient, and disease recurrence was observed after 2 months. Due to the small size of specimens, gene test

Table I Medication of apatinib combined with erlotinib

\begin{tabular}{|c|c|c|c|c|}
\hline $\begin{array}{l}\text { Time periods } \\
\text { (in 2016) }\end{array}$ & $\begin{array}{l}\text { Time duration } \\
\text { (days) }\end{array}$ & $\begin{array}{l}\text { Apatinib dose } \\
\text { (mg) }\end{array}$ & $\begin{array}{l}\text { Erlotinib dose } \\
\text { (mg) }\end{array}$ & Reasons for dosage adjustment \\
\hline From March 17 to April 6 & 20 & 250 qd & $150 \mathrm{qd}$ & Start from the minimum dosage \\
\hline From April 7 to May 6 & 30 & $\begin{array}{l}250 \text { qod from April } 7 \\
500 \text { qod from April } 8\end{array}$ & $150 \mathrm{qd}$ & $\begin{array}{l}\text { Gradually add the dosage to guarantee the } \\
\text { curative effect }\end{array}$ \\
\hline From May 7 to 12 & 6 & Suspension & $150 \mathrm{qd}$ & $\begin{array}{l}\text { Fear of bleeding caused by quickly emerging } \\
\text { cavitation; hypertension }\end{array}$ \\
\hline From May 13 to 28 & 16 & 250 per $72 \mathrm{~h}$ & $150 \mathrm{qd}$ & $\begin{array}{l}\text { Resume taking the medicine because of the } \\
\text { increasing mass }\end{array}$ \\
\hline From May 29 to June 23 & 10 & 250 per $48 \mathrm{~h}$ & $150 \mathrm{qd}$ & Return to original dosage \\
\hline From June 14 to June 23 & 10 & $\begin{array}{l}250 \\
2 \text { days consecutively } \\
\text { and I day cessation }\end{array}$ & $150 \mathrm{qd}$ & Gradually return to original dosage \\
\hline From June 24 to July 8 & 16 & $250 \mathrm{qd}$ & 150 qd & Gradually return to original dosage \\
\hline From July 9 to August 24 & 46 & 250 qd & 150 qod & Maintenance treatment; anorexia, feebleness; VTE \\
\hline From August 25 to 31 & 7 & Suspension & Suspension & $\begin{array}{l}\text { Extensive subcutaneous hemorrhage; disease } \\
\text { progression to death }\end{array}$ \\
\hline
\end{tabular}




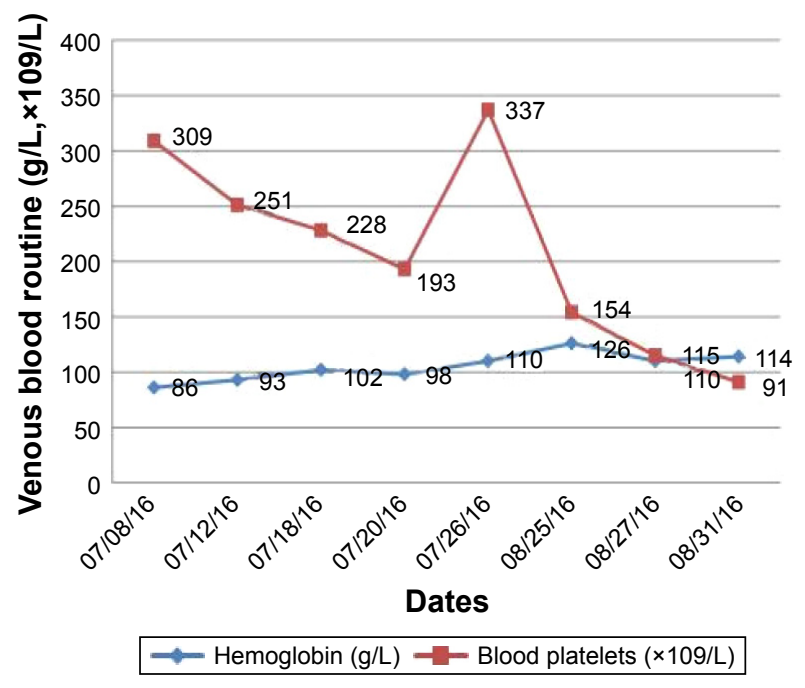

Figure 4 The venous blood routine from anticoagulant therapy.

cannot be performed. However, considering the high rate of EGFR mutation in Asians, erlotinib was chosen as the second-line therapy without EGFR test when the patient refused to receive chemotherapy. Evaluation after application of erlotinib for 1 month was effective, suggesting the patient might be harboring EGFR mutation. After 5 months, drug resistance occurred, which in most patients after 9-13 months of treatment. ${ }^{7-9}$ The following therapeutic strategy is to develop third-generation drugs against T790M resistance mutation or to investigate new combination strategies to overcome this resistance. However, plasma test (cfDNA) was not introduced by China-Japan Friendship Hospital then. Neither was osimertinib against T790M-positive NSCLC in Mainland China. ${ }^{10}$ Herein, the patient would like to accept combination targeted treatment.

Angiogenesis plays a significant role in carcinoma growth and metastasis, while the VEGF signaling is the key pathway in vascular endothelial cells. ${ }^{11}$ In vitro study has verified that the co-treatment with anti-VEGF therapy could enhance antitumor activity of anti-EGFR therapy. ${ }^{12}$ Targeting angiogenesis by inhibition of VEGFs was shown to be effective for lung cancer ${ }^{13}$ including bevacizumab and apatinib.

Bevacizumab, a humanized monoclonal antibody against VEGF, has been recommended as the first-line therapy for lung adenocarcinoma until disease progression in NCCN Guidelines. ${ }^{14}$ In the randomized open-label phase III BEVERLY study, the combined treatment of erlotinib and bevacizumab was significantly better than erlotinib alone in terms of PFS in patients with nonsquamous NSCLC with activating EGFR mutations, suggesting the possible combination strategy to combat EGFR-TKI resistance. ${ }^{15}$ Furthermore, a systematic review and meta-analysis validated the benefits of PFS and overall response rate from the second-line cotreatment of NSCLC with bevacizumab and erlotinib. ${ }^{16}$ Case reports also indicated that the combination of bevacizumab and erlotinib enhanced the efficacy against central nervous system metastasis, especially leptomeningeal carcinomatosis after failure of erlotinib. ${ }^{17,18}$ Like bevacizumab, apatinib is a kind of vascular endothelial growth inhibitor, while the oral agent ensures the ease in use and is affordable to the patient, considering the patient's choice of having targeted treatment at home. Apatinib, is also a novel oral anti-angiogenesis agent, potently suppressing the c-kit and c-src, and inhibiting the cellular phosphorylation of VEGFR-2, c-kit, and PDGFR beta, ${ }^{2}$ achieved certain antitumor drug effect and clinical benefits. Median overall survival and PFS were also significantly improved in the apatinib group compared with the placebo group in phase III trial. ${ }^{5}$ In light of the above, it should be considered to use apatinib to combat TKI resistance. The patient benefited another 5.1-month PFS after confronting erlotinib resistance.

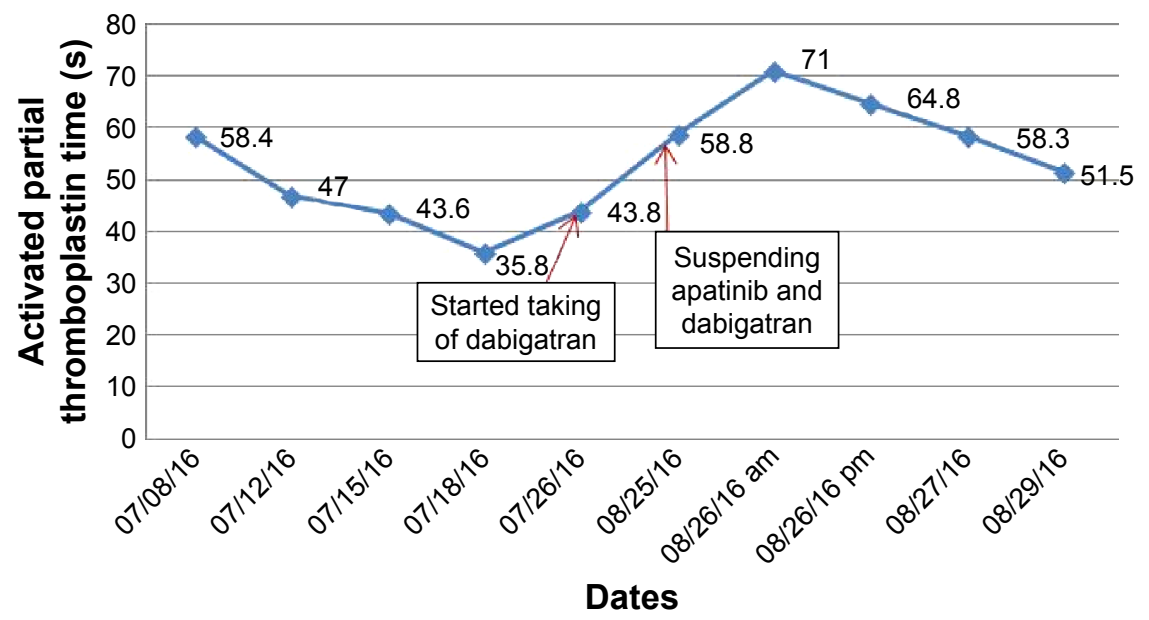

Figure $\mathbf{5}$ The activated partial thromboplastin time from anticoagulant therapy. 


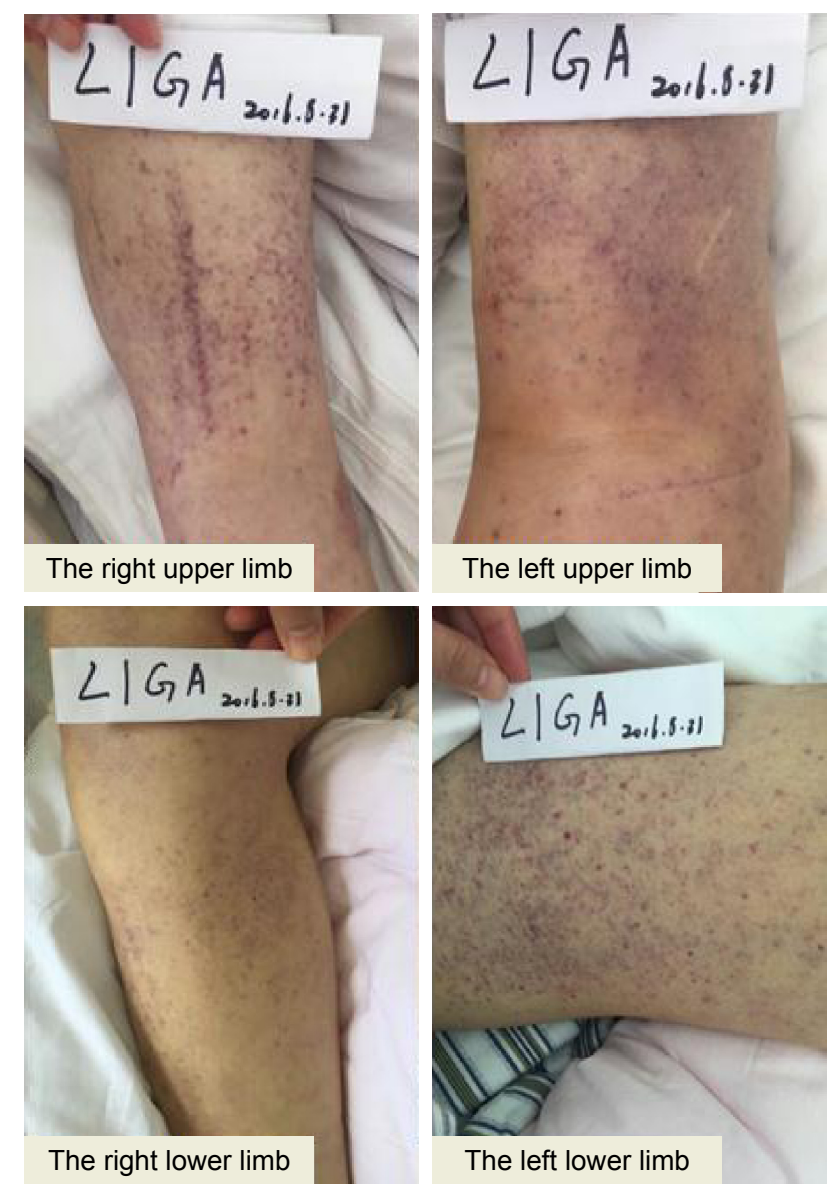

Figure 6 The subcutaneous hemorrhage in limbs on August 31, 2016.

During the treatment of apatinib, the patient suffered from AEs, causing dosage adjustment. In phase II trial, the dosing regimen of $850 \mathrm{mg}$ once daily was recommended for the treatment of gastric cancer. ${ }^{4}$ Hypertension, hand-food syndrome, and proteinuria were known to be the most common AEs of antiangiogenic agents in the phase I/II/III trials, and the incidence of hematologic toxicities was low. ${ }^{4-6} \mathrm{After}$ this patient taking the minimum dosage of $250 \mathrm{mg}$ once daily for $\sim 1$ month, thoracic CT and cranial MRI showed marked benefits, although facing the challenge of hypertension as well as bleeding when dabigatran was used. The dosage was constantly adjusted in accordance with the disease changes, reaching a maximum of $250 \mathrm{mg}$ once daily. Dosing of apatinib requires adjustments in different cancers and populations to optimize efficacy and benefits to patients.

Cancer patients are more vulnerable to VTE. A recent study showed that a cumulative VTE incidence was as low as $3.2 \%$ over a follow-up of 2.5 years, a much lower risk compared with new diagnosis. ${ }^{19}$ The patient had cancerrelated VTE after 23 months of cancer diagnosis. According to the current guidelines, patients are usually treated with anticoagulation for at least 3 months, ${ }^{20}$ especially those with cancer-related thrombosis who would benefit a lot from anticoagulation therapy, while the therapeutic regimen should adjust with cancer status. ${ }^{21}$ The current standard of care in patients with cancer and VTE is LMWH..$^{22}$ Fraxiparine was given for 18 days and dabigatran was given for 27 days as outpatient treatment for the patient before wide-spread subcutaneous hemorrhage occurred.

Dabigatran, a new oral anticoagulant, is the direct thrombin inhibitor. In the sister trials RE-COVER and RE-COVER II, ${ }^{23,24}$ treatment with dabigatran for VTE in patients with cancer offers a similarly effective, safe, and more convenient alternative to VKA in terms of fixed drug dosing. Renal and hepatic functions of this patient were found normal after dabigatran was taken; however, subcutaneous hemorrhage could not be well controlled. The patient had lower blood platelet and higher APTT. No medication can effectively neutralize the anticoagulant effect in China at present. Probably, apatinib might worsen the bleeding by blocking new blood vessel formation or having interactions with dabigatran. Current studies showed that apatinib was metabolized primarily by CYP $3 \mathrm{~A} 4 / 5 ;{ }^{25}$ however, dabigatran was metabolized by esterases in the plasma and liver without significant involvement of CYP3A4 ${ }^{26,27}$ They might have a low potential for drug-drug interactions though other more specific profile is unknown.

\section{Conclusion}

Apatinib may be a recommendation for lung cancer patients and such studies are ongoing. To date, no evidence has been published on the use of apatinib against EGFR-TKI resistance in patients. Our study indicates that apatinib could be a potential option to combat EGFR-TKI resistance. However, further studies are required to verify these findings as well as more exploration on pharmacological dosage and AEs is needed.

\section{Acknowledgments}

This case report was supported by China-Japan Friendship Hospital and Beijing Chest Hospital. We would like to thank Professor Qing Wu (English Department, School of Humanities, Beijing University of Chinese Medicine, Beijing, China) for English language editing. The authors thank the patient and his wife.

\section{Author contributions}

YP, HC, ZL, and DL was responsible for collection and assembly of the patient's data. YP, FL, YS, HD, YQ, and QL performed data analysis and literature search. All authors 
contributed toward data analysis, drafting and revising the paper and agree to be accountable for all aspects of the work.

\section{Disclosure}

The authors report no conflicts of interest in this work.

\section{References}

1. Torre LA, Bray F, Siegel RL, Ferlay J, Lieuleut J, Jemal A. Global Cancer Statistics, 2012. CA Cancer J Clin. 2015;65(2):87-108.

2. Tian S, Quan H, Xie C, et al. YN968D1 is a novel and selective inhibitor of vascular endothelial growth factor receptor-2 tyrosine kinase with potent activity in vitro and in vivo. Cancer Sci. 2011;102(7):1374-1380.

3. Ding J, Chen X, Gao Z, et al. Metabolism and pharmacokinetics of novel selective vascular endothelial growth factor receptor-2 inhibitor apatinib in humans. Drug Metab Dispos. 2013;41(6):1195-1210.

4. Li J, Qin S, Xu J, et al. Apatinib for chemotherapy-refractory advanced metastatic gastric cancer: results from a randomized, placebo-controlled, parallel-arm, phase II trial. J Clin Oncol. 2013;31(26):3219-3225.

5. Li J, Qin S, Xu J. Trial of apatinib in patients with chemotherapyrefractory advanced or metastatic adenocarcinoma of the stomach or gastroesophageal junction. J Clin Oncol. 2016;34(13):1448.

6. Li J, Zhao X, Chen L, et al. Safety and pharmacokinetics of novel selective vascular endothelial growth factor receptor-2 inhibitor YN968D1 in patients with advanced malignancies. BMC Cancer. 2010;10:529.

7. Zhou C, Wu YL, Chen G, et al. Erlotinib versus chemotherapy as first-line treatment for patients with advanced EGFR mutation-positive non-smallcell lung cancer (OPTIMAL, CTONG-0802): a multicentre, pen-label, randomised, phase 3 study. Lancet Oncol. 2011;12(8):735-742.

8. Rosell R, Carcereny E, Gervais R, et al. Erlotinib versus standard chemotherapy as first-line treatment for European patients with advanced EGFR mutation positive non-small-cell lung cancer (EURTAC): a multicentre, open-label, randomized phase 3 trial. Lancet Oncol. 2012;13: 239-246.

9. Wu YL, Zhou C, Liam CK, et al. Firstline erlotinib versus gemcitabine/ cisplatin in patients with advanced EGFR mutation positive non-smallcell lung cancer: analyses from the phase III, randomized, open-label, ENSURE study. Ann Oncol. 2015;26:1883-1889.

10. Mok TS, Wu YL, Ahn MJ, et al. Planchard D. Osimertinib or PlatinumPemetrexed in EGFR T790M-Positive Lung Cancer. $N$ Engl J Med. 2017;376:629-640.

11. Chatterjee S, Heukamp LC, Siobal M, et al. Tumor VEGF: VEGFR2 autocrine feed forward loop triggers angiogenesis in lung cancer. $J$ Clin Invest. 2013;123(4):1732-1740.

12. Li H, Takayama K, Wang S, et al. Addition of bevacizumab enhances antitumor activity of erlotinib against non-small cell lung cancer xenografts depending on VEGF expression. Cancer Chemother Pharmacol. 2014;74(6):1297-1305.
13. Folkman J. Angiogenesis in cancer, vascular, rheumatoid and other disease. Nat Med. 1995;1(1):27-31.

14. Ettinger DS, Wood DE, Akerley W, et al. NCCN Guidelines Insight: nonsmall cell lung cancer, version 4. 2016. J Natl Compr Canc Netw. 2016; 14(3):255-264.

15. Gridelli C, Rossi A, Ciardiello F, et al. BEVERLY: rationale and design of a randomized open-label phase III trial comparing bevacizumab plus erlotinib versus erlotinib alone as first-line treatment of patients with EGFR-mutated advanced nonsquamous non-small-cell lung cancer. Clin Lung Cancer. 2016;17(5):461-465.

16. Zhang S, Mao XD, Wang HT, Cai F, Xu J. Efficacy and safety of bevacizumab plus erlotinib versus bevacizumab or erlotinib alone in the treatment of non-small-cell lung cancer: a systematic review and meta-analysis. BMJ Open. 2016;6(6):e011714.

17. Ariyasu R, Horiike A, Koyama J, et al. Efficacy of bevacizumab and erlotinib combination for leptomeningeal carcinomatosis after failure of erlotinib. Anticancer Drugs. In press 2017.

18. Sakata Y, Kawamura K, Shinqu N, Ichikado K. Erlotinib plus bevacizumab as an effective treatment for leptomeningeal metastases from EGFR mutation-positive non-small cell lung cancer. Lung Cancer. 2016;99:120-122.

19. Prandoni P, Casiglia E, Piccioli A, et al. The risk of cancer in patients with venous thromboembolism does not exceed that expected in the general population after the first 6 months. J Thromb Haemost. 2010;8(5): $1126-1127$.

20. O'Connell C. How I treat incidental pulmonary embolism. Blood. 2015;125(12):1877-1882.

21. Kearon C, Akl EA. Duration of anticoagulant therapy for deep vein thrombosis and pulmonary embolism. Blood. 2014;123(12):1794-1801.

22. Lee AY, Levine MN, Baker RI, et al. Low-molecular weight heparin versus a coumarin for the prevention of recurrent venous thromboembolism in patients with cancer. N Engl J Med. 2003;349(2): 146-153.

23. Schulman S, Kearon C, Kakkar AK, et al. Dabigatran versus warfarin in the treatment of acute venous thromboembolism. NEngl J Med. 2009; 361(24):2342-2352.

24. Schulman S, Kakkar AK, Goldhaber SZ, et al. Treatment of acute venous thromboembolism with dabigatran or warfarin and pooled analysis. Circulation. 2014;129(7):764-772.

25. Ding JF, Chen XY, Gao ZW, et al. Metabolism and pharmacokinetics of novel selective vascular endothelial growth factor receptor-2 inhibitor apatinib in humans. Drug Metab Dispos. 2013;41(6):1195-1210.

26. Scaglione F. New oral anticoagulants: comparative pharmacology with vitamin K antagonists. Clin Pharmacokinet. 2013;52(2):69-82.

27. Short NJ, Connors JM. New oral anticoagulants and the cancer patient. Oncologist. 2014;19(1):82-93.
OncoTargets and Therapy

\section{Publish your work in this journal}

OncoTargets and Therapy is an international, peer-reviewed, open access journal focusing on the pathological basis of all cancers, potential targets for therapy and treatment protocols employed to improve the management of cancer patients. The journal also focuses on the impact of management programs and new therapeutic agents and protocols on

\section{Dovepress}

patient perspectives such as quality of life, adherence and satisfaction. The manuscript management system is completely online and includes a very quick and fair peer-review system, which is all easy to use. Visit http://www.dovepress.com/testimonials.php to read real quotes from published authors. 